Appeared in the Journal of Chemical Physics, 125, 194508

\title{
Time dependent diffusion in a disordered medium with partially absorbing walls: A perturbative approach
}

\author{
Jiang Qian ${ }^{1,2}$ and Pabitra N. Sen ${ }^{2}$, \\ ${ }^{1}$ Lyman Laboratory of Physics, Harvard University, Cambridge, MA 02138, USA \\ ${ }^{2}$ Schlumberger-Doll Research, Ridgefield, CT 06877-4108, USA
}

(Dated: November 10, 2018)

\begin{abstract}
We present an analytical study of the time dependent diffusion coefficient in a dilute suspension of spheres with partially absorbing boundary condition. Following Kirkpatrick (J. Chem. Phys. $76,4255)$ we obtain a perturbative expansion for the time dependent particle density using volume fraction $f$ of spheres as an expansion parameter. The exact single particle $t$-operator for partially absorbing boundary condition is used to obtain a closed form time-dependent diffusion coefficient $D(t)$ accurate to first order in the volume fraction $f$. Short and long time limits of $D(t)$ are checked against the known short-time results for partially or fully absorbing boundary conditions and longtime results for reflecting boundary conditions. For fully absorbing boundary condition the long time diffusion coefficient is found to be $D(t)=5 a^{2} /\left(12 f D_{0} t\right)+O\left(\left(D_{0} t / a^{2}\right)^{-2}\right)$, to the first order of perturbation theory. Here $f$ is small but non-zero, $D_{0}$ the diffusion coefficient in the absence of spheres, and $a$ the radius of the spheres. The validity of this perturbative result is discussed.
\end{abstract}




\section{INTRODUCTION}

There are numerous processes in biology, physics, geophysics, chemical engineering and materials science where a diffusing species reacts upon contact with the surface of another phase (typically a solid) and the effects of simultaneous diffusion and reaction are important. Studying the time-dependence of concentration of the species reacting with a porous host via diffusion, which goes back to Smoluchowsky $(1913)^{1}$, remains an important problem both for practical and theoretical reasons: for reviews, see Weiss ${ }^{2}$, Kayser and Hubbard ${ }^{3}$, Fixman $^{4}$, Torquato $^{5}$, deGennes ${ }^{6}$. Heterogeneous catalysis, transport and absorption of nutrients and drugs are well known examples. Another example is relaxation of Nuclear magnetization of interstitial fluid by interaction with walls in the NMR experiments. The connection between surface relaxation and NMR goes back to the seminal 1951 paper "On nuclear relaxation in gases by surface catalysis" by Bloch ${ }^{7}$ himself. NMR Relaxation is now routinely used in numerous settings ranging from clinical $\mathrm{MRI}^{8}$ to geological explorations for energy ${ }^{9}$. The purpose of this paper is to investigate time-dependence of the diffusion coefficient of molecules confined in a well-connected porous host material with reactive walls.

Diffusion measurements on surviving number of random walkers can give deep insight into this complex process of diffusion-relaxation. Experimentally observing the dynamics (diffusion) of molecules reveals a distinct set of information that cannot be obtained from the traditional relaxation (i.e. number of surviving walkers), measurements ${ }^{11}$. The complex porous systems, in whose interstices the fluid reside, have in general, convoluted and extensive well connected pores. The time dependence of the rate of relaxation in a well connected geometry which does not have arbitrarily large voids (see below) is not fully understood, not to mention diffusion. The relaxation processes are well understood for isolated cells or pores (isolated cavities), where an eigen-mode decomposition ${ }^{12,13,14}$ of the diffusion equation with partially absorbing boundary condition is possible. Disentangling the complexity due to the combined effects of disorder, diffusion and decay remains a major hurdle in connected porous media ${ }^{11}$.

A general theory of time-dependent diffusion and relaxation in porous media does not exist. At short-times, there are asymptotic results that are robust for all boundary conditions and arbitrary geometry ${ }^{11,15,16}$. For long-time, however, no general technique exists, except for simple models of a dilute periodic array of spheres with weak absorption ${ }^{17}$. In random 
systems, a dilute suspension of randomly placed spheres is most well studied. For reflecting (non-absorbing) boundary condition de Swiet and $\mathrm{Sen}^{18}$ worked out a perturbation expansion based on Bixon and Zwanzig ${ }^{19}$ that gives short and long time behavior of the time dependent diffusion coefficient.

The main goal of this paper is to extend these results ${ }^{18}$ for partially absorbing boundary conditions. For partially absorbing boundary conditions perturbation expansions fail at long times ${ }^{10,17}$. The long time behavior of diffusion in such a system has not been previously addressed either analytically or numerically. Decay in particle number density with fully absorbing boundary condition has been studied extensively, see for example the review by Weiss $^{2}$. The simplest effective medium approach of Smoluchowsky gives an exponential decay for the total number of surviving particles $N(t)$. Bixon and Zwanzig pioneered the perturbative approach which gave a surprisingly slow power law decay. This discrepancy has triggered an intense interest in the problem. Kirkpatrick ${ }^{10}$ perfected the perturbation theory, and he showed that by summing a select group of terms (the most divergent) this power law tail was removed and the exponential decay was recovered. However, the higher order terms in the perturbation series diverge as well and are difficult to re-sum and one cannot rule out long-time power law tails ${ }^{10}$.

Later, allowing arbitrarily large fluctuations in local density, Grassberger and Proccacia, Kayser and Hubbard, and others ${ }^{3,20,21}$ conclusively showed that the long time limiting behavior of $N(t)$ in such system shows a non-perturbative behavior: in a $d$-dimension system $N(t)$ shows a stretched exponential decay $N(t) \rightarrow \exp \left[-t^{d /(d+2)}\right]$ as $t \rightarrow \infty .{ }^{24}$ This result cannot be captured by any (known) re-summation of the perturbation expansion. However three issues are important- (i) real systems do not have large voids and this stretched exponential result is of limited use (ii) Fixman ${ }^{4}$ has argued that the crossover from the effective medium theory (simple exponential decay in time) to the exact limiting behavior (i.e. the stretched exponential) can be extraordinarily slow, i.e. the breakdown occurs at so long a time that $N(t)$ is too small to be of any experimental relevance. (iii) we are interested in $D(t)$ and not, per se, in $N(t) . D(t)$ is given by mean-square displacement divided by $N(t)$, there is no known rigorous bound for this ratio. It is possible that perturbative expansion can give a reasonable answer for $D(t)$. We note in passing that Grassberger and Proccacia ${ }^{20}$ give a heuristic argument that $D(t)$ approaches zero as $t^{-d /(d+2)}$ at long times. Even such a heuristic conjecture does not exist for partially absorbing systems nor for systems that do 
not have large fluctuations in density.

Therefore, we may argue that, for experimentally accessible times $t$, the perturbation expansion describes diffusion reasonably well and this is what we proceed to carry out. In the next section II we carry out a perturbation expansion for a dilute suspension of randomly placed spheres. We obtain an analytical expression of the approximate Green's function from first order terms of that expansion. From the Green's function we find the simple short and long time limiting behavior of time dependent diffusion coefficient $D(t)$.

\section{ANALYTICAL RESULTS FOR SUSPENSION OF SPHERE}

In this section we study the problem of diffusion in dilute system with the partially absorbing boundary condition analytically. Only dilute systems are amenable to analytical methods. We will address the range of validity of such methods at the end of this section.

Let us consider now diffusion of particles around a randomly distributed dilute suspension of $N$ spheres, all of which of a given radius $a$, centered at positions $\vec{R}_{i}, 1 \leq i \leq N$. The density $C(\vec{r}, t)$ of particles is governed by diffusion equation

$$
\frac{\partial C(\vec{r}, t)}{\partial t}=D_{0} \nabla^{2} C(\vec{r}, t)
$$

and the partially absorbing boundary condition is imposed:

$$
\begin{gathered}
D_{0} \vec{n}_{i} \cdot \nabla C\left(\left|\vec{r}-\vec{R}_{i}\right|=a, t\right)=\rho C\left(\left|\vec{r}-\vec{R}_{i}\right|=a, t\right), \\
C\left(\left|\vec{r}-\vec{R}_{i}\right|<a, t\right)=0,
\end{gathered}
$$

for $i$ from 1 to $N$, where $\vec{n}_{i}$ is the unit vector normal to the $i$ th sphere, pointing outward. The initial condition is

$$
C(\vec{r}, t=0)=C_{0}(\vec{r})
$$

Following Kirkpatrick ${ }^{10}$ we go into the Laplace domain for time and the Fourier domain for space and express density in terms of t-operator of individual sphere as scattering center,

$$
\begin{aligned}
& C(\vec{q}, \epsilon)=G_{0}(q) C_{0}(\vec{q})+\sum_{i=1}^{N} G_{0}(q) T_{i}(\vec{q}) C_{i}(\vec{q}, \epsilon), \\
& C_{i}(\vec{q}, \epsilon)=G_{0}(q) C_{0}(\vec{q})+\sum_{j \neq i}^{N} G_{0}(q) T_{j}(\vec{q}) C_{j}(\vec{q}, \epsilon) .
\end{aligned}
$$


Here $\epsilon$ is the reciprocal Laplace variable for time and $\vec{q}$ is the reciprocal Fourier variable for space and $G_{0}(q)=\left(\epsilon+D q^{2}\right)^{-1}$ is the free particle Green's function, i.e. diffusion in unbounded space, and $T_{i}$ is the Fourier space $t$-operator of a single sphere centered at $R_{i}$. Combining Eq. (5) and Eq. (6) we have the following perturbative expansion for the density $^{10}$ :

$$
\begin{array}{r}
C(\vec{q}, \epsilon)=\left\{G_{0}(q)+\sum_{i=1}^{N} G_{0}(q) T_{i}(\vec{q}) G_{0}(q)+\sum_{i=1}^{N} \sum_{j \neq i}^{N} G_{0} T_{i}(\vec{q}) G_{0}(q) T_{j}(\vec{q}) G_{0}(q)+\right. \\
\left.\sum_{i=1}^{N} \sum_{j \neq i}^{N} \sum_{k \neq j}^{N} G_{0} T_{i}(\vec{q}) G_{0}(q) T_{j}(\vec{q}) G_{0}(q) T_{k}(\vec{q}) G_{0}(q)+\cdots\right\} C_{0}(\vec{q}),
\end{array}
$$

This equation has the same form of the binary collision expansion in kinetic theory of gases ${ }^{10}$. This naive expansion encounters a problem at long time ${ }^{10,22}$ and it is better to sum the series to avoid such difficulties ${ }^{10,19}$. The averaged results reads:

$$
C(\vec{q}, \epsilon)=\left\{\epsilon+D_{0} q^{2}-\sum_{i=1}^{\infty} n^{i} \int \prod_{j=1}^{i} d \vec{R}_{i} B_{i}\left(\vec{q}, \vec{R}_{1} \ldots \vec{R}_{i}, \epsilon\right)\right\}^{-1} C_{0}(q)
$$

Here $n=N / V$ is the number of spheres per unit volume and $B_{i}\left(\vec{q}, \vec{R}_{i}, \epsilon\right)$ represents the "self energy" operator for multiple scattering and includes correlation effects of averaging of $i$ scattering center. For example, when Eq. (8)is expanded, the operator form gives

$$
\begin{gathered}
\int d \vec{R}_{1} B_{1}\left(\vec{q}, \vec{R}_{1}, \epsilon\right)=\int d \vec{R}_{1} T_{1}(\vec{q}), \\
\int d \vec{R}_{1} d \vec{R}_{2} B_{2}\left(\vec{q}, \vec{R}_{1}, \vec{R}_{2}, \epsilon\right)=\int d \vec{R}_{1} \int d \vec{R}_{2}\left\{T_{1}(\vec{q}) G_{0}(q) T_{2}(\vec{q}) G_{0}(q) T_{1}(\vec{q})+\right. \\
\left.T_{1}(\vec{q}) G_{0}(q) T_{2}(\vec{q}) G_{0}(q) T_{1}(\vec{q}) G_{0}(q) T_{2}(\vec{q})+\cdots\right\} .
\end{gathered}
$$

As usual, here $T_{i}$ is the $t$-operator of a single sphere at position $\vec{R}_{i}$ and the integrals are averaging over trap positions. In this paper we confine ourself to the effect of the first term of the expansion, i.e. we include multiple scattering from a single sphere to all orders, but do not consider correlation effects of multiple spheres.

As required by translational invariance, the dependence of the $t$-operator on the position of sphere $\vec{R}_{i}$ is very simple:

$$
t_{i}\left(\vec{q}, \overrightarrow{q^{\prime}}, \epsilon, \vec{R}_{i}\right)=\exp \left[-i \vec{R}_{i} \cdot\left(\vec{q}-\overrightarrow{q^{\prime}}\right)\right] t\left(\vec{q}, \overrightarrow{q^{\prime}}, \epsilon\right)
$$


Therefore the lowest order position averaged $t$-operator simply gives a factor of $(2 \pi)^{3} \delta^{3}\left(\vec{q}-\overrightarrow{q^{\prime}}\right)$ and we only need the diagonal element of the $t$-operator $\langle t\rangle=t(q, q, \epsilon)$.

Transformed into Laplace space the diffusion equation reads:

$$
\left(D_{0} \nabla^{2}-\epsilon\right) C(\vec{r}, \epsilon)=-C_{0}(\vec{r})
$$

The boundary condition in Eq. (2) becomes, in Laplace domain:

$$
\lambda a \vec{n}_{i} \cdot \nabla C(\vec{r}, \epsilon)-C(\vec{r}, \epsilon)=0 ; \quad \lambda=\frac{D_{0}}{\rho a}
$$

on the surface $\left|\vec{r}-\vec{R}_{i}\right|=a$. Here we introduced a dimensionless parameter $\lambda=D_{0} / \rho a$ as a measure of strength of absorption on surface. $\lambda=0$ corresponds to totally absorbing boundary condition and $\lambda=\infty$ corresponds to reflecting boundary condition.

It is convenient to choose the initial condition $C_{0}(\vec{r})=\delta^{3}(\vec{r}-\vec{r} \prime)$, which when Fourier transformed against $\vec{r} \prime$ ) reads:

$$
C_{0}(\vec{r}, \vec{q} \prime, \epsilon)=\frac{e^{i \vec{q} \cdot \vec{r}}}{(2 \pi)^{3}}
$$

A special solution for inhomogeneous equation (12) is

$$
C_{\text {special }}(\vec{r}, \vec{q} \prime, \epsilon)=\frac{1}{(2 \pi)^{3}} \frac{e^{i \vec{q} \cdot \vec{r}}}{\epsilon+D_{0} q^{\prime 2}}
$$

and the general solution of the homogeneous equation satisfying boundary condition that density to be finite at infinity is:

$$
C_{\text {general }}(\vec{r}, \epsilon)=\sum_{i=0}^{\infty} b_{i} P_{i}\left(\cos \theta^{\prime}\right) \kappa_{i}\left(\frac{\left|\vec{r}-\vec{R}_{i}\right| \sqrt{s}}{a}\right),
$$

where $\kappa_{i}(x)$ is the $i$ th order modified spherical Bessel function and $P_{i}(x)$ is the $i$ th order Legendre polynomial. $\theta^{\prime}$ is the angle between $\vec{q}^{\prime}$ and $\vec{r}-\vec{R}_{i}$ and we introduce dimensionless parameter $s=\epsilon a^{2} / D_{0}$ to replace $\epsilon$.

Choosing constants $b_{i}$ of Eq. (16) to make a linear combination with Eq. (15) that satisfy boundary condition Eq. (13) we have for $\left|\vec{r}-\vec{R}_{i}\right|>a$ :

$C(\vec{r}, \vec{q}, \epsilon)=\frac{1}{(2 \pi)^{3}} \frac{e^{i \vec{q} \cdot \vec{R}_{i}}}{\epsilon+D_{0} q^{2}}\left[e^{i \vec{q} \cdot \cdot\left(\vec{r}-\vec{R}_{i}\right)}-\sum_{n=0}^{\infty}(2 n+1) i^{n} P_{n}\left(\cos \theta^{\prime}\right) \frac{\lambda\left(q^{\prime} a\right) j_{n}^{\prime}\left(q^{\prime} a\right)-j_{n}\left(q^{\prime} a\right)}{\lambda \sqrt{s} \kappa_{n}^{\prime}(\sqrt{s})-\kappa_{n}(\sqrt{s})} \kappa_{n}\left(\frac{\left|\vec{r}-\vec{R}_{i}\right| \sqrt{s}}{a}\right)\right]$ 
where $j_{n}(x)$ is the $n$th order spherical Bessel function.

Fourier transforming Eq. (17) with respect to $r$ we can easily obtain the single particle $t$ operator:

$$
\begin{gathered}
t(\vec{q}, \vec{q}, \epsilon)=-\frac{4 \pi a D_{0} e^{i\left(\vec{q}-\vec{q}^{\prime}\right) \cdot \vec{R}_{i}}}{(2 \pi)^{3}}\left\{\frac{s+k^{2}}{|\vec{k}-\vec{k}|} j_{1}(|\vec{k}-\vec{k} \prime|)+\sum_{n=0}^{\infty}(2 n+1) P_{n}(\cos \theta) \times\right. \\
\left.\frac{\lambda k^{\prime} j_{n}^{\prime}\left(k^{\prime}\right)-j_{n}\left(k^{\prime}\right)}{\lambda \sqrt{s} \kappa_{n}^{\prime}(\sqrt{s})-\kappa_{n}(\sqrt{s})}\left[\sqrt{s} j_{n}(k) \kappa_{n+1}(\sqrt{s})-k j_{n+1}(k) \kappa_{n}(\sqrt{s})\right]\right\} ; \quad \vec{k}=\vec{q} a ; \vec{k} \prime=\vec{q} \prime a
\end{gathered}
$$

Here we made the obvious choice for dimensionless momentum $\vec{k}=\overrightarrow{q a}$ and $\vec{k} \prime=\vec{q} \prime a$ and $\theta$ is the angle between $\vec{k}$ and $\vec{k} /$. This form for the $t$-operator manifestly obeys Eq. (11). Setting $\vec{k}=\vec{k} \prime$ we easily obtain the diagonal element of the $t$-operator

$$
\langle t\rangle=-\left(4 \pi a D_{0}\right)\left\{\frac{1}{3}\left(s+k^{2}\right)+\sum_{n=0}^{\infty}(2 n+1) \frac{\lambda k j_{n}^{\prime}(k)-j_{n}(k)}{\lambda \sqrt{s} \kappa_{n}^{\prime}(\sqrt{s})-\kappa_{n}(\sqrt{s})}\left[\sqrt{s} j_{n}(k) \kappa_{n+1}(\sqrt{s})-k j_{n+1}(k) \kappa_{n}(\sqrt{s})\right]\right\}
$$

To obtain the effective diffusion coefficient, we expand Eq. (19) to order $k^{2}$ and obtain the following leading order behavior:

$$
\langle t\rangle=-\left(4 \pi a D_{0}\right)\left\{\frac{1}{3}\left(s+k^{2}\right)+\frac{1+\sqrt{s}}{1+\lambda+\lambda \sqrt{s}}-k^{2}\left[\frac{-1+(3+\sqrt{s}) \lambda)+(1+2 \sqrt{s}+s) \lambda^{2}}{3(1+\lambda+\lambda \sqrt{s})(1+\sqrt{s}+(2+2 \sqrt{s}+s) \lambda}\right]\right\}
$$

Taking the lowest order in Eq. (8), combining with Eq. (9) and definition of Green's function we have $\langle G\rangle=\left(G_{0}^{-1}-\frac{N}{V}\langle t\rangle\right)^{-1}$. Substituting from Eq. (20) we have, again to the order of $k^{2}$ :

$$
\langle G\rangle=\frac{a^{2}}{D_{0}}\left\{s+f\left[s+\frac{3(1+\sqrt{s})}{(1+\lambda+\lambda \sqrt{s})}\right]\right\}^{-1}\left\{1-k^{2}\left[\frac{1+f\left(1-\frac{-1+(3+\sqrt{s}) \lambda+(1+\sqrt{s})^{2} \lambda^{2}}{(1+\lambda+\lambda \sqrt{s}(1+\sqrt{s}+\lambda(2+2 \sqrt{s}+s))}\right)}{s+f\left(s+\frac{3(1+\sqrt{s})}{(1+\lambda+\lambda \sqrt{s})}\right)}\right]\right\} .
$$

Here we define the volume fraction of the spheres $f=\frac{4 \pi a^{3} N}{3 V}$. This generalizes Kirkpatrick's ${ }^{10}$ Eq. (A5) to finite absorption. To obtain $D(t)$ notice that for the system with partially 
absorbing boundary condition:

$$
\left\langle r^{2}\right\rangle=-6 a^{2} \frac{\lim _{k \rightarrow 0} \partial G(k, t) / \partial k^{2}}{\lim _{k \rightarrow 0} G(k, t)} .
$$

The numerator is the inverse Laplace transform of the following expression:

$$
\frac{\left[1+f\left(1-\frac{-1+(3+\sqrt{s}) \lambda+(1+\sqrt{s})^{2} \lambda^{2}}{(1+\lambda+\lambda \sqrt{s})(1+\sqrt{s}+\lambda(2+2 \sqrt{s}+s))}\right)\right]}{\left[s+f\left(s+\frac{3(1+\sqrt{s})}{(1+\lambda+\lambda \sqrt{s})}\right)\right]^{2}} .
$$

The denominator, that is the number of surviving particles $N(\tilde{t})$, is the inverse Laplace transform of $G(k=0)$ :

$$
\frac{1}{s+f\left[s+\frac{3(1+\sqrt{s})}{(1+\lambda+\lambda \sqrt{s})}\right]} .
$$

Performing the inverse Laplace transform of the above equation, we obtain for the denominator, the total number of surviving particles:

$$
N(\tilde{t})=\frac{1}{(1+f)\left(r_{1}-r_{2}\right)\left(r_{1}-r_{3}\right)\left(r_{2}-r_{3}\right) \lambda}\left[e^{r_{1}^{2} \tilde{t}} r_{1}\left(r_{2}-r_{3}\right)\left(r_{1} \lambda+\lambda+1\right) \operatorname{erfc}\left(-r_{1} \sqrt{\tilde{t}}\right)+c . p .\right],
$$

here $\tilde{t}=D_{0} t / a^{2}, \operatorname{erfc}(x)=1-\operatorname{erf}(x)$ is the complementary error function, c.p. denotes cyclical permutation $r_{1} \rightarrow r_{2}, r_{2} \rightarrow r_{3}$ and $r_{3} \rightarrow r_{1}$, and $r_{1}, r_{2}, r_{3}$ are the roots of the cubic equation:

$$
(1+\lambda) f r^{3}+(1+f+\lambda f) r^{2}+3 f r+3 f=0 .
$$

To check this with the known results we look at several limits where the forms of $N(t)$ are simple.

First consider the short time limit $\tilde{t} \ll 1, i$. e., $t \ll a^{2} / D_{0}$. There are two subcases. If the boundary is nearly reflecting, specifically $\sqrt{s} \gg 1 / \lambda$, i.e. $\rho \ll \sqrt{D_{0} / t}$, the results is particularly simple:

$$
N(\tilde{t})=1-f-\frac{3 f \tilde{t}}{\lambda}+\frac{4 f \tilde{t}^{3 / 2}}{\sqrt{\pi} \lambda^{2}}+O\left(\tilde{t}^{5 / 2}\right) .
$$

Here $1-f$ factor comes from the excluded volume of the spheres. The first term agrees with equation (A9) of Mitra et. al. ${ }^{16}$, which is only to the first order in $f$.

In the other extreme, when $\rho \gg \sqrt{D_{0} / t}$, that is the nearly fully absorbing boundary condition, the short time total number of particle becomes insensitive to $\rho$ :

$$
N(\tilde{t})=1-f-\frac{\left(6 f-12 f^{2}\right) \sqrt{\tilde{t}}}{\sqrt{\pi}}+\left(-3 f+15 f^{2}\right) \tilde{t}+O\left(\tilde{t}^{3 / 2}\right),
$$


The first term in turns agrees with equation (A8) of Mitra et. al. ${ }^{16}$, again to the first order in $f$.

Next consider the long-time limits. The total particle number decays, in the long time limit, $\tilde{t} \gg 1$, i.e., $t \gg a^{2} / D_{0}$, for fully absorbing boundary condition:

$$
N(\tilde{t})=\frac{1}{6 f \sqrt{\pi}} \tilde{t}^{-3 / 2}+\frac{2-f+2(1+f) \lambda}{12 f^{2} \sqrt{\pi}} \tilde{t}^{-5 / 2}+O\left(\tilde{t}^{-7 / 2}\right)
$$

The first term is twice the perturbative result in Bixon and Zwanzig ${ }^{19}$. Recall that the exact non-perturbative result of Grassberger and Procaccia ${ }^{20}$ gives a stretched exponential decay. Also note the $1 / f$ dependence, for small but finite $f$.

Finally consider the diffusion coefficient with partially absorbing boundary conditions. Results from the inverse Laplace transform of the numerator in Eq. (23) is too complex to be reproduced fully here, so we summarize the result in a simpler form:

$$
\lim _{k \rightarrow 0} \frac{\partial G(k, \tilde{t})}{\partial k^{2}}=\sum_{i=1}^{3} \sum_{j=1}^{2} A_{i j} I_{j}\left(r_{i}, \tilde{t}\right)+\sum_{i=4}^{5} A_{i 1} I_{1}\left(r_{i}, \tilde{t}\right)
$$

In this equation, $r_{1-3}$ are the same three roots of the cubic equation Eq. (26) and $r_{4,5}=$ $\left(-1-2 \lambda \pm \sqrt{1-4 \lambda^{2}}\right) / 2 \lambda$ are the two roots of the quadratic equation. And the constants $A_{i j}$, which depends on $r_{1}$ through $r_{5}$ above as well as $\lambda$ and $f$, are the coefficients of partial fractions of Eq. (23):

$$
\begin{aligned}
& \frac{\left[1+f\left(1-\frac{-1+(3+\sqrt{s}) \lambda+(1+\sqrt{s})^{2} \lambda^{2}}{(1+\lambda+\lambda \sqrt{s})(1+\sqrt{s}+\lambda(2+2 \sqrt{s}+s))}\right)\right]}{\left[s+f\left(s+\frac{3(1+\sqrt{s})}{(1+\lambda+\lambda \sqrt{s})}\right)\right]^{2}} \\
& =\sum_{i=1}^{5} \frac{A_{i 1}}{\sqrt{s}-r_{i}}+\sum_{i=1}^{3} \frac{A_{i 2}}{\left(\sqrt{s}-r_{i}\right)^{2}} .
\end{aligned}
$$

$I_{1,2}$ are given by the following expression obtained by inverse Laplace transform of the partial fractions:

$$
\begin{aligned}
& I_{1}\left(r_{i}, \tilde{t}\right)=\frac{1}{\sqrt{\pi \tilde{t}}}+r_{i} e^{r_{i}^{2} t} \operatorname{erfc}\left(-r_{i} \sqrt{\tilde{t}}\right) \\
& I_{2}\left(r_{i}, \tilde{t}\right)=\frac{2 r_{i}^{2} \sqrt{\tilde{t}}}{\sqrt{\pi}}+\left(1+2 r_{i}^{2}\right) r_{i} e^{r_{i}^{2} \tilde{t}} \operatorname{erfc}\left(-r_{i} \sqrt{\tilde{t}}\right)
\end{aligned}
$$

At short time $t \ll a^{2} / D_{0}$ and for the reflecting boundary condition, using the fact $N(\tilde{t})=$ $1-f$, we recover the results of Eq. (14)of De Swiet and $\operatorname{Sen}^{18}$ :

$$
D(\tilde{t})=D_{0}\left[1-\frac{4 f \sqrt{\tilde{t}}}{3 \sqrt{\pi}}+O(\tilde{t})\right]
$$


For the fully absorbing boundary condition at short time, we expand Eq. (30) to obtain:

$$
\lim _{k \rightarrow 0} \frac{\partial G(k, \tilde{t})}{\partial k^{2}}=1-\frac{20 f}{3 \sqrt{\pi}} \tilde{t}^{3 / 2}+O\left(\tilde{t}^{2}\right) .
$$

This combined with $N(t)$ obtained in Eq. (28), gives to the first order of $f$

$$
D(t) / D_{0}=1-\frac{2 f \sqrt{\tilde{t}}}{3 \sqrt{\pi}}+O\left(\tilde{t}^{2}\right),
$$

which is consistent with the result in Mitra et. al. ${ }^{16}$ Eq. $(\mathrm{A} 11)^{25}$.

Much more interesting is the case of fully absorbing boundary condition at long time $t \gg a^{2} / D_{0}$. There the numerator of Eq. (23) reduced to $\{f(f(6 f+29)+19)+5\}\left\{18 f^{2}(f+\right.$ 1) $(f+4) \sqrt{\pi}\} \tilde{t}^{-3 / 2}+O\left(\tilde{t}^{-5 / 2}\right)$. Keeping $f$ to first order and combining with Eq. (22) and the result for $N(\tilde{t})$ Eq. (29) we obtain the surprising result:

$$
D(\tilde{t}) / D_{0}=\frac{5}{12 f} \tilde{t}^{-1}+O\left(\tilde{t}^{-2}\right),
$$

that is, within perturbation theory, the long time diffusion coefficient $D(t)$ of the dilute suspension of spheres with absorbing boundary condition approaches zero as $1 / t$. Notice that $D(\tilde{t})$ is inversely dependent on volume fraction $f$ as is $N(\tilde{t})$. There is a subtle order of limit: though we are taking an asymptotic expansion for small $f$, the limit where Eq. (37) is valid is for a small yet fixed $f$ but $\tilde{t} \rightarrow \infty$, i.e. $\tilde{t} \gg 1 / f$, where the limit is well defined in the equation.

Next consider some known results. For diffusion in a straight tube with fully absorbing wall, the separation of variables can be used to obtain exact results. The number of particles $N(t)$ will decay exponentially to zero with time, but $D(t) \rightarrow D_{0} / 3$ as $t \rightarrow \infty$ because the particles may diffuse along the axis of the tube. Any long tube-like open pore with slowly changing diameter will also have finite diffusion coefficient. Furthermore a periodic array of spheres with fully absorbing boundary condition always have finite diffusion coefficient at long time at any volume fraction. In fact there is no known connected system, excluding isolated-pore like structures (e.g. in the Lifshitz limit ${ }^{20}$ where the absorbers form a cavity), with any amount of surface relaxation, which has a vanishing long time diffusion coefficient. Intuitively a dilute random suspension of spheres is not likely to be an exception. So the perturbative result of $1 / t$ behavior of $D(t)$ may indicate a rapid drop of diffusion coefficient at a certain range of $t$ but is unlikely to give the correct true long time behavior. 


\section{CONCLUSION}

The $1 / t$ time dependence for $D(t)$ given by the perturbative result for random suspension is peculiar, as is the $1 / t^{3 / 5}$ behavior given by the heuristic arguments of Grassberger and Procaccia $^{20}$. While analytical perturbative method considered here give deep insight and give correct result for short-time limit, we suspect that perturbative results for the long time behavior of $D(t)$ will break down just as the perturbative result fails for total number of surviving particles $N(t)$ at long times. In view of the findings of Fixman ${ }^{4}$, it will be interesting to examine the perturbative results for $D(t)$ against numerical results with a hope that for the time-regime of experimental interest, perturbative results will suffice.

\section{Acknowledgments}

The authors would like to thank B. I. Halperin and A. M. Turner for a careful reading of the manuscript. JQ would like to acknowledge the people of the NMR group at Schlumberger-Doll Research for their kind hospitality. This work was also supported in part by the National Science Foundation grant DMR-99-81283.

1 M. V. Smoluchowsky, Phys. Z., 17, 557 (1916)

2 G. H. Weiss, J. Stat. Phys.,42,3 (1986); Aspects and Applications of the Random Walk, North Holland Press, Amsterdam (1994).

3 R. F. Kayser and J. B. Hubbard J. Chem. Physics, 80, 1127 (1984)

4 M. Fixman, Phys. Rev. Letts., 52,791 (1984)

5 AR Kansal and S. Torquato, J. Chem. Physics, 116, 10589 (2002)

6 P-G. deGennes, C. R. Acad Sc. Paris,295,1061 (1982)

7 F. Bloch Phys. Rev. 83, 1062 ?1063 (1951)

8 D. M. Grant and R. K. Harris Encyclopedia of Nuclear Magnetic Resonance Ed D. M. Grant and R. K. Harris, Wiley. New York (1996)

9 R. L. Kleinberg, "Well Logging" in Encyclopedia of Nuclear Magnetic Resonance, Ed D. M. Grant and R. K. Harris, Wiley. New York (1996), p 4960.

10 T. R. Kirkpatrick, J. Chem. Phys. 76, 4255 (1982). 
11 P. N. Sen, Concepts in Magnetic Resonance, 23 A (1), 1 (2004)

12 K. R.Brownstein and C. E. Tarr, Importance of classical diffusion in NMR studies of water in biological cells, Phys. Rev. A 19 ,2446 ( 1979).

13 L. J. Zielinski, Y.-Q. Song, S. Ryu, and P. N. Sen, Characterization of coupled pore systems from the diffusion eigenspectrum, J. Chem. Phys. 117, 5361 (2002)

14 L. Marinelli, M.D. Hürlimann, and P.N. Sen, Modal analysis of q-space - relaxation correlation experiments, J. Chem. Phys., 118, 8927, (2003)

15 P. P. Mitra, P. N. Sen, L. M. Schwartz and P. Le Doussal, Physical Rev. Lett, 65, 3555, (1992);

16 P. P. Mitra, P. N. Sen and L. M. Schwartz, Phys. Rev. B 47, 8565 (1993). Eq. (30) has severe typographical mistakes which can be fixed by multipying the right side by $\left(\kappa D_{0}+\rho\right) /\left(\kappa D_{0}-\rho\right)$ Also, a factor of $S / V_{p}$ is missing from the last term in Eq. (29)

17 P. N. Sen, L. M. Schwartz, P. P. Mitra, and B. I. Halperin. Phys. Rev. B, 49:215, 1994.

18 T. M. de Swiet and P. N. Sen, J. Chem. Phys., 104, 206, 1996.

19 M. Bixon,and R.Zwanzig, J. Chem. Phys. 75, 2354 (1981).

20 P. Grassberger and I. Procaccia. The long time properties of diffusion in a medium with static traps. J. Chem. Phys. 776281 (1982)

21 M. D. Donsker M. D. and S. R. S. Varadhan, Asympototic evaluation of certain Markov processes expectations for large time. IV. Comm. Pure Appl. Math., 36:183-212. 1983

22 M. Ernst, L. Haines, and J. R. Dorfman, Rev. Mod. Phys. 41, 296 (1969).

23 R. Zwanzig, Phys. Rev. 129, 468 (1963).

24 This result also holds for partially absorbing boundary condition ${ }^{3}$

25 Here we take $N(t=0)=N_{0}(1-f)$ instead $N_{0}$ to be consistent ease the comparison with results in $^{16}$ 